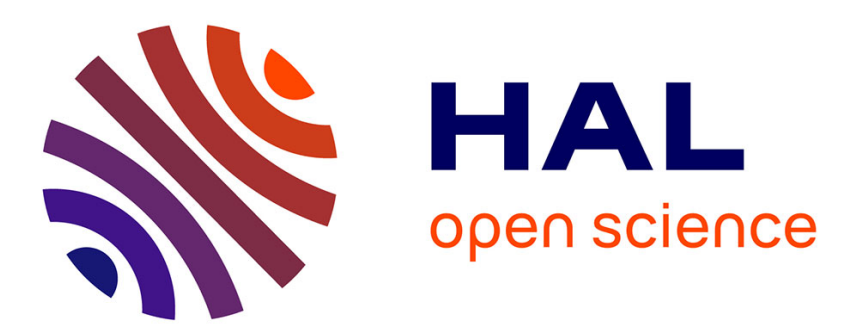

\title{
Ultrasound-Stimulated Phase-Change Contrast Agents for Transepithelial Delivery of Macromolecules, Toward Gastrointestinal Drug Delivery
}

Samantha M Fix, Bhanu P Koppolu, Anthony Novell, Jared Hopkins, Thomas M Kierski, David A Zaharoff, Paul A Dayton, Virginie Papadopoulou

\section{- To cite this version:}

Samantha M Fix, Bhanu P Koppolu, Anthony Novell, Jared Hopkins, Thomas M Kierski, et al.. Ultrasound-Stimulated Phase-Change Contrast Agents for Transepithelial Delivery of Macromolecules, Toward Gastrointestinal Drug Delivery. Ultrasound in Medicine \& Biology, 2019, 45 (7), pp.1762-1776. 10.1016/j.ultrasmedbio.2019.02.004 . hal-03324571

\section{HAL Id: hal-03324571 \\ https://hal.science/hal-03324571}

Submitted on 23 Aug 2021

HAL is a multi-disciplinary open access archive for the deposit and dissemination of scientific research documents, whether they are published or not. The documents may come from teaching and research institutions in France or abroad, or from public or private research centers.
L'archive ouverte pluridisciplinaire HAL, est destinée au dépôt et à la diffusion de documents scientifiques de niveau recherche, publiés ou non, émanant des établissements d'enseignement et de recherche français ou étrangers, des laboratoires publics ou privés. 
1 Ultrasound-stimulated phase-change contrast agents for transepithelial delivery of 2 macromolecules, towards gastrointestinal drug delivery

4 Samantha M. Fix ${ }^{\mathrm{a}}$, Bhanu P. Koppolu ${ }^{\mathrm{b}}$, Anthony Novell ${ }^{\mathrm{c}}$, Jared Hopkins ${ }^{\mathrm{b}}$, Thomas M. Kierski ${ }^{\mathrm{b}}$ David A. 5 Zaharoff ${ }^{\mathrm{b}}$, Paul A. Dayton ${ }^{\mathrm{a}, \mathrm{b}}$, Virginie Papadopoulou ${ }^{\mathrm{b}, *}$

6

7 a Eshelman School of Pharmacy, University of North Carolina, Chapel Hill, NC 27599, USA

8 b Joint Department of Biomedical Engineering, The University of North Carolina and North Carolina State

9 University, Chapel Hill, North Carolina 27599, USA

10 c IR4M, Université Paris-Saclay, CNRS UMR 8081, 91401 Orsay, France

$12 *$ Corresponding author:

13 Virginie Papadopoulou

14 papadopoulou@unc.edu

15 919-843-8492

$16 \quad 310$ Taylor Hall

17109 Mason Farm Rd

18 Chapel Hill, NC 27599 


\section{ABSTRACT}

22 The gastrointestinal (GI) tract presents a notoriously difficult barrier for macromolecular drug delivery,

23 especially for biologics. Herein, we demonstrate that ultrasound-stimulated phase change contrast agents

24 (PCCAs) can transiently disrupt Caco-2 monolayers and improve the transepithelial transport of a 25 macromolecular model drug. With ultrasound treatment in the presence of PCCAs, we achieved a 26 maximum of $44 \pm 15 \%$ transepithelial delivery of $70 \mathrm{kDa}$ FITC-dextran, compared to negligible delivery 27 through sham control monolayers. Among all tested rarefactional pressures $(300-600 \mathrm{kPa})$, dextran 28 delivery efficiency was consistently greatest at $300 \mathrm{kPa}$. To explore this unexpected finding, we quantified 29 stable and inertial cavitation energy generated by various ultrasound exposure conditions. In general, 30 lower pressures resulted in more persistent cavitation activity over 30 second exposure times, which may 31 explain the enhanced dextran delivery efficiency. Thus, a unique advantage of using low boiling point 32 PCCAs for this application is that the same low-pressure pulses can be used to induce vaporization and 33 provide maximal delivery.

\section{KEYWORDS}

36 Ultrasound; Transepithelial Delivery; Acoustic Droplet Vaporization; Phase-change contrast agents;

37 Nanodroplet 
INTRODUCTION

The gastrointestinal (GI) tract has evolved to efficiently breakdown food products, allow nutrient

40 uptake, and simultaneously provide a robust defense against ingested toxins and pathogens (Barrett 2012,

41 Schoellhammer, et al. 2016). This presents a complex barrier for both local and systemic drug delivery 42 through the GI tract, particularly for biologics (e.g., therapeutic peptides, proteins, and nucleic acids) 43 (Goldberg and Gomez-Orellana 2003). The first hurdle is maintaining drug stability in the degradative environment. This often requires specialized formulations to protect the active pharmaceutical ingredient from (1) the harsh and fluctuating acidic $\mathrm{pH}$ and (2) an array of degradative species and enzymes (Moss, et al. 2017). If drug integrity is maintained, the second hurdle is permeability. The GI tract is blanketed with mucus that traps and protects the underlying epithelium from foreign particles (Cone 2009). Furthermore, GI epithelial cells are linked by tight junctions that restrict paracellular transport of large molecules (> $200 \mathrm{Da}$ ), and the hydrophilic nature of most biologics limits passive transcellular diffusion (Goldberg and Gomez-Orellana 2003). Due to these challenges, generally only small molecule drugs can be delivered through the GI tract.

Therapeutic ultrasound has recently been proposed as a universal method to physically enhance GI permeability to macromolecular drugs (Schoellhammer, et al. 2017, Schoellhammer, et al. 2016, Schoellhammer, et al. 2017, Schoellhammer, et al. 2015, Schoellhammer and Traverso 2016). This technique involves colonic insertion of an ultrasound probe with co-administration of a medicated enema (Schoellhammer and Traverso 2016). Low-frequency ( $\leq 100 \mathrm{kHz})$ ultrasound exposure for one minute or less has been shown to significantly enhance macromolecule delivery into surrounding GI tissue 58 (Schoellhammer, et al. 2017, Schoellhammer, et al. 2017). This technology has the potential to transform

59 the treatment landscape of several GI diseases including ulcerative colitis, cancers of the GI tract, 60 infection, and Crohn's disease, and offers the following unique benefits (Schoellhammer, et al. 2017, 
61 Schoellhammer, et al. 2016, Schoellhammer, et al. 2015). First, rapid delivery limits concerns of drug degradation by intestinal enzymes, eliminating the need for complex protective formulations. Second, this technique may allow for local GI delivery of biologic drugs previously restricted to intravenous administration (including peptides, proteins, monoclonal antibodies, nucleic acids), which would expand therapeutic options for GI diseases. Finally, concentrated local delivery has the potential to enhance drug efficacy and reduce systemic toxicities.

The mechanism of ultrasound-mediated drug delivery involves in situ generation and acoustic excitation of vapor cavities near a biological barrier through the process of cavitation (Lentacker, et al. 2014, Miller, et al. 2002, Schoellhammer and Traverso 2016). When a fluid is subjected to ultrasound of sufficient energy, vapor cavities are created (cavitation). These bubbles oscillate with the passing acoustic wave (stable cavitation) and cause flow disturbances in the surrounding fluid, and this 'microstreaming' exerts shear stress on nearby structures (Kooiman, et al. 2014, Pereno, et al. 2018). With sufficient acoustic amplitude, the bubble becomes unstable, violently collapses (inertial cavitation), and creates a fluid microjet towards nearby boundaries. Stresses exerted by these physical phenomena transiently enhance the permeability of biological barriers (Coussios, et al. 2016, Escoffre and Bouakaz 2018, Kooiman, et al. 2014, Miller, et al. 2002, Postema, et al. 2005). In addition to enhanced permeability, acoustic cavitation and radiation force enhance the transport of molecules through newly permeabilized membranes (Bhatnagar, et al. 2014).

The likelihood of ultrasound to induce cavitation is related to the wave's mechanical index (MI) which is defined as the peak rarefactional pressure (in MPa) divided by the square root of the center 81 frequency (in MHz) (Apfel and Holland 1991, Bhatnagar, et al. 2014). To achieve ultrasound-mediated 82 drug delivery, low frequencies $(\leq 100 \mathrm{kHz})$ and consequently high MIs $(>2)$ are often needed 83 (Schoellhammer, et al. 2017, Schoellhammer, et al. 2017, Schoellhammer, et al. 2015). However, 
introducing exogenous cavitation nuclei into the acoustic field substantially lowers the energy required to induce cavitation-mediated bioeffects as initial bubble formation is no longer required (Bhatnagar, et al. 2014). This can be achieved through the use of microbubble-based ultrasound contrast agents. Microbubbles are 1-10 $\mu \mathrm{m}$ gas-filled particles stabilized by a lipid, protein or polymer shell (Sirsi and Borden 2009). When subjected to ultrasound, they oscillate and/or collapse, providing biological permeabilization similar to that described above, but with acoustic parameters in line with what is employed for clinical diagnostic imaging (i.e. with MIs less than the U.S. Food and Drug Administration's limit of 1.9) (Delalande, et al. 2011, Lentacker, et al. 2014). Indeed, drug delivery by this technique can 92 be achieved with diagnostic ultrasound imaging equipment, which provides the added benefit of simultaneous ultrasound image guidance (Kotopoulis, et al. 2013).

The use of ultrasound-stimulated microbubbles for drug delivery has been studied extensively for the disruption of vascular barriers (Martin and Dayton 2013, Sennoga, et al. 2017). For applications such as opening the blood brain barrier (Burgess and Hynynen 2014, Wu, et al. 2017, Zhao, et al. 2013) or enhancing vascular permeability within solid tumors (Mullick Chowdhury, et al. 2017, Qin, et al. 2016), microbubbles are administered intravenously and are stimulated with an external ultrasound source. We envision an adaptation of this technology for GI drug delivery, which would involve co-administration of contrast agents with a medicated enema in the colon followed by ultrasound stimulation using an endoscopic probe. With the use of contrast agents, we believe that higher frequencies and lower pressures may be used compared to the previously described, contrast-free methods of ultrasound-mediated GI drug delivery. This approach may theoretically localize bioeffects more precisely, as permeabilization would only occur where contrast agents are present (e.g., at the GI wall). This would reduce concerns of unsuppressed cavitation activity outside the desired treatment area (e.g., surrounding tissues). 
As a first step towards this goal, we studied the dynamics of epithelial monolayer disruption and recovery in vitro using phase-change ultrasound contrast agents (PCCAs) stimulated with $1.0 \mathrm{MHz}$ ultrasound pulses. PCCAs are liquid perfluorocarbon-filled particles that can be vaporized into acoustically active microbubbles with the application of an ultrasound pulse of sufficient amplitude. In the liquid state, PCCAs are characterized by nanometer-range size distributions (100-300 nm) that may allow more thorough permeation through the GI mucus mesh compared to microbubbles $(0.5-10 \mu \mathrm{m}$ (Sirsi and Borden 2009)). Once vaporized, PCCAs form microbubbles several microns in diameter that can be utilized to enhance drug delivery.

Typically, PCCAs are created using perfluorocarbons with bulk boiling points near body temperature (e.g. dodecafluoropentane, b.p. $=29^{\circ} \mathrm{C}$ ). These agents require acoustic pressures in the range of 3-6 MPa to initiate vaporization (Burgess and Porter 2015, Williams, et al. 2013), which may induce undesired tissue bioeffects (e.g., cell lysis or tissue heating) for applications where transient drug delivery is desired. We have developed a low boiling point formulation of PCCAs filled with octofluoropropane (OFP), which has a bulk boiling point of $-36.7^{\circ} \mathrm{C}$. These agents can be vaporized with peak negative pressures as low as $300 \mathrm{kPa}$ (at $1.0 \mathrm{MHz}$ ) (Fix, et al. 2017) and with clinically available hardware. We believe that this low boiling point PCCA formulation is ideal for GI drug delivery applications where transient permeabilization is desired without permanent tissue damage.

The primary objective of this study is to demonstrate the potential of using ultrasound-stimulated PCCAs to cause transient disruption of confluent colorectal adenocarcinoma (Caco-2) epithelial monolayers and enhance the permeation of a model macromolecular drug. This represents a first step towards the goal of in vivo GI drug delivery applications. 

PCCAs were generated as previously described (Sheeran, et al. 2011, Sheeran, et al. 2012). Briefly, purity, Avanti Polar Lipids, Alabaster, AL, USA) were combined in a 9:1 molar ratio and solubilized in a phosphate-buffered saline (PBS)-based solution (1× PBS: $11.9 \mathrm{mM}$ phosphates, $137 \mathrm{mM}$ solium chloride, $2.7 \mathrm{mM}$ potassium chloride) containing $15 \%$ propylene glycol $(v / v)(\geq 99.5 \%$ purity, Fisher Chemical, Waltham, MA, USA) and 5\% glycerol ( $v / v)(\geq 99.5 \%$ purity, Fisher Chemical, Waltham, MA, USA) for a final combined concentration of $1.0 \mathrm{mg} / \mathrm{mL}$. Aliquots $(1.5 \mathrm{~mL})$ of this solution were dispensed into 3.0 $\mathrm{mL}$ glass vials, the headspace air was replaced with octofluoropropane (OFP) gas $(99.8 \%$ purity, FluoroMed, Round Rock, TX, USA), and microbubbles were generated via shaking for 45 seconds in a 140 VialMix device, which operates at a frequency of $4530 \pm 100$ oscillations per minute (Bristol-Myers141 Squibb, New York, NY, USA). Finally, PCCAs were formed via microbubble condensation through simultaneous cooling and pressurization of the microbubble suspension (Sheeran, et al. 2012). The size distribution of resultant PCCAs was characterized using a NanoSight NS500 (Malvern Instruments, Westborough, MA, USA) (detection capability 50-2000 nm) (n=3 representative vials). to spontaneous vaporization due to the very low boiling point of OFP. However, we previously showed that stimulating cells with ultrasound and OFP-filled PCCAs below the vaporization threshold of the PCCAs does not cause barrier permeabilization and does not result in substantial cavitation activity (Fix, et al. 2017). This indicates that spontaneous vaporization alone likely does not contribute a substantial enough bubble population to influence drug delivery outcome. Furthermore, in vivo imaging studies from 
PCCAs, substantial vaporization can be achieved in a controllable manner via ultrasound-induced vaporization (Sheeran, et al. 2015).

\section{PCCA vaporization captured via high-speed optical microscopy}

PCCA vaporization events were captured at a range of acoustic settings using high-speed optical microscopy, using a previously described experimental set-up (Sheeran, et al. 2013). Briefly, a temperature-controlled water bath $\left(37^{\circ} \mathrm{C}\right)$ was mounted on an inverted microscope with a $100 \times$ water immersion objective (Olympus IX71, Center Valley, PA, USA), and the microscope was interfaced with a high-speed camera to capture vaporization events (FastCam SA1.1, Photron USA, Inc., San Diego CA, USA).

First, a calibrated needle hydrophone (HNA-0400, Onda Corp., Sunnyvale, CA, USA) was aligned to the optical focus of the microscope and subsequently used to characterize the peak negative pressure (PNP) of a 1.0 MHz unfocused piston transducer (IP0102HP, Valpey Fisher Corp., Hopkinton, MA,

USA). The transducer was excited with sinusoidal pulses generated by an arbitrary function generator (AFG3021, Tektronix, Inc., Beaverton, OR, USA) and amplified approximately $55 \mathrm{~dB}$ with a power amplifier (A500, ENI, Rochester, NY, USA). After the transducer was calibrated, the hydrophone was replaced with a $200-\mu \mathrm{m}$ inner diameter microcellulose tube (Spectrum Labs, Inc., Rancho Dominguez, CA, USA), which was also aligned to the optical focus of the microscope. This process ensured that contents of the microcellulose tube that were visible in the microscope focus would experience the acoustic pressure calibrated at that location. The microscope field of view was $130 \mu \mathrm{m} \times 130 \mu \mathrm{m}$. PCCAs were diluted 1:4 in PBS and perfused through the microcellulose tube. Injection of the PCCAs was followed by a brief waiting period to allow particles in the field of view to become nearly 
vaporization is a pressure-threshold dependent phenomenon, independent of pulse length when submillisecond pulses are used (Fabiilli, et al. 2009, Lo, et al. 2007). Therefore, to determine an approximate pressure threshold for vaporization, PCCAs were exposed to single 20-cycle acoustic pulses with PNPs of $100,200,300,400,500$, or $600 \mathrm{kPa}$.

A synchronization pulse from the function generator was used to trigger the high-speed camera and initiate video recording at 500 frames per second. Videos were set to begin recording just before the triggered ultrasound pulse, which allowed complete PCCA vaporization events within the optical field of view to be captured. Videos were reviewed offline using ImageJ to determine whether or not vaporization was achieved at the various acoustic excitation parameters (National Institutes of Health, Bethesda, MD, USA).

\section{Cell culture}

Human colorectal adenocarcinoma (Caco-2) cells were chosen as an in vitro model of intestinal absorption. When Caco-2 monolayers are grown to confluence, they differentiate, developing polarity and tight junctions between cells. This accurately mimics the human intestinal epithelial barrier, making Caco2 permeability testing a reliable tool for predicting in vivo intestinal absorption of various compounds (van Breemen and Li 2005).

Mycoplasma-free caco-2 cells were purchased from the American Type Culture Collection (ATCC) and cultured using minimum essential medium without L-glutamine (Corning Inc., Corning, NY, USA) supplemented with $100 \mathrm{U} / \mathrm{mL}$ penicillin/streptomycin, $1 \times$ non-essential amino acid supplement (NEAA), $1 \times$ sodium pyruvate, $1 \times$ L-glutamate, and $20 \%$ heat-inactivated fetal bovine serum $(\mathrm{FBS})$ (VWR, Radnor, PA, USA) in a humidified, $37^{\circ} \mathrm{C}$ incubator with $5 \% \mathrm{CO}_{2}$ atmosphere. Low passage number (passage 15) Caco-2 cells were seeded at 150,000 cells per well onto Transwell supports with 0.4 $\mu \mathrm{m}$ pores (12-well plate, $12 \mathrm{~mm}$ membrane diameter, $1.12 \mathrm{~cm}^{2}$ cell growth area, Corning Inc., Corning, 
NY, USA) and allowed to grow for approximately 10 days to form confluent monolayers. Monolayer integrity was regularly monitored via the measurement of transepithelial electrical resistance (TEER)

(World Precision Instruments, Sarasota, FL, USA). Monolayers were used for permeability experiments after TEER values had reached at least $500 \mathrm{Ohm}\left(560 \mathrm{Ohm} \cdot \mathrm{cm}^{2}\right)$, which is similar to TEER values reported in the literature for this model (Kudsiova and Lawrence 2008, Srinivasan, et al. 2015). Furthermore, above this threshold, macromolecular tracers (e.g., the $70 \mathrm{kDa}$ dextran used here) are unable to permeate through the Caco-2 monolayers.

\section{Ultrasound alignment strategy}

Caco-2 monolayers were sonicated from the apical side using a $1.0 \mathrm{MHz}$ unfocused piston transducer (diameter $=8 \mathrm{~mm},-6 \mathrm{~dB}$ 'focal spot' $=\sim 6 \mathrm{~mm}$ diameter, IP0102HP, Valpey Fisher Corp., Hopkinton, MA, USA). A custom water bath was designed to (1) facilitate consistent alignment of the transducer above the center of each well and (2) reduce acoustic reflections from the base of the well plate (Figure 1). The $14 \mathrm{~cm} \times 10 \mathrm{~cm} \times 8.5 \mathrm{~cm}(\mathrm{~L} \times \mathrm{W} \times \mathrm{H})$ water bath was created using $3.2 \mathrm{~mm}$ acrylic sheets. For each experiment, a 12-well culture plate was placed on an internal ledge within the bath, providing coupling between the bottom of the well plate and the pre-heated $37^{\circ} \mathrm{C}$ water below. Water was maintained at $37^{\circ} \mathrm{C}$ for the duration of the experiment by placing the apparatus on a heated plate. Coupling between the well plate and water was designed to minimize acoustic reflections that would have otherwise occurred at the air-plastic interface, although we do note that it is unlikely that this strategy entirely eliminated reflections and standing waves. A lid for the water bath was created with $1.0 \mathrm{~cm}$-diameter circular holes centered above each well. This served as a method to hold the ultrasound transducer centered within each well at a consistent height above the cells $(6 \mathrm{~mm})$. The full design of this water bath can be found in the supplemental materials, which includes complete instructions for fabrication (available in Mendeley Data repository online). 


\section{Ultrasound-mediated FITC-dextran delivery through epithelial monolayers}

Twelve-well plates containing confluent Caco-2 monolayers on Transwell ${ }^{\circledR}$ supports were

positioned in the custom water bath as described above. Complete cell media (400 $\mu 1)$ containing PCCAs

$\left(0.5 \mu \mathrm{l} ; 1.75 \times 10^{9}\right.$ particles $/ \mathrm{mL}$; roughly matching the circulating concentration of OFP-PCCAs used as vascular ultrasound contrast agents (Sheeran, et al. 2015)) and $70 \mathrm{kDa}$ FITC-dextran (50 $\mu \mathrm{g}$ total; 0.125 $\mathrm{mg} / \mathrm{mL}$; similar in concentration to prior reports of colonic delivery of fluorescent dextran in mice (Schoellhammer, et al. 2017)) was added to the donor chamber (apical side) of a well to simulate absorption from the intestinal lumen. The $70 \mathrm{kDa}$ dextran was chosen to model macromolecular drugs typically unable to pass the GI epithelial barrier. Subsequently, the $1.0 \mathrm{MHz}$ transducer was placed in center of the well and excited with amplified sinusoidal signals from an arbitrary function generator (AFG3021C, Tektronics, Inc., Beaverton, OR, USA and 3100LA Power Amplifier, ENI, Rochester, NY, USA) (Figure 1). A pulse repetition frequency of $5000 \mathrm{~Hz}$ and exposure time of 30 seconds was used for all conditions. Pulse lengths of 20 cycles ( $10 \%$ duty cycle) or 40 cycles ( $20 \%$ duty cycle) were employed, and peak negative pressures were varied from 300 to $600 \mathrm{kPa}$. Control samples were exposed to (1) sham treatment (without PCCAs or ultrasound), (2) PCCAs only, and (3) ultrasound only (using the highest energy condition of $600 \mathrm{kPa}$ and 40 cycle pulse length). A summary of the conditions tested can be found in Table 1, including experimental replicates for each group. time points after treatment and replaced with cell media: $\sim 5 \mathrm{~min}, 3 \mathrm{hr}, 8 \mathrm{hr}, 24 \mathrm{hr}, 48 \mathrm{hr}$, and $72 \mathrm{hr}$. Dextran concentration was determined via fluorescence intensity (excitation: $485 \mathrm{~nm}$, emission: $528 \mathrm{~nm}$ ) using a Cytation 5 Plate Reader (BioTek, Winooski, VT, USA). Percent dextran delivery was calculated based on the mass initially added to the donor compartment, correcting for the mass removed through sampling at 
each time point and considering $100 \%$ to be the receiving well concentration if equilibrium had been established between both compartments.

TEER values were recorded at the same time points and used to monitor monolayer disruption and recovery dynamics. For all experiments, monolayers with a TEER value of at least $560 \mathrm{Ohms} \cdot \mathrm{cm}^{2}$ were considered intact, and monolayers below this threshold were considered disrupted. "Time to monolayer recovery" was calculated as a summary metric to quantify the degree of monolayer disruption caused by sonication. For monolayers that recovered by the last measurement time point ( 72 hours), time to recovery was calculated as the time that TEER values remained below $560 \mathrm{Ohms} \cdot \mathrm{cm}^{2}$, using a linear interpolation between measured time points. Monolayers that never dropped below $560 \mathrm{Ohms} \cdot \mathrm{cm}^{2}$ therefore had a time to monolayer recovery of 0 hours. reduced to $2000 \mathrm{~Hz}$. Unfortunately, hardware limitations prevented us from saving data with a pulse repetition frequency of $5000 \mathrm{~Hz}$ to match the cell experiments. We do not believe that this discrepancy

\section{Detection of cavitation signals and their persistence during 30 second ultrasound exposures}

Cavitation is thought to be the main mechanical driver of biological barrier permeabilization with ultrasound. As such, we were interested in characterizing the generation and persistence of stable and inertial cavitation energy generated by ultrasound-stimulated PCCAs during 30 second exposures. We detected cavitation signals using a method adapted from our previous work (Fix, et al. 2017). PCCAs $\left(1.75 \times 10^{9}\right.$ particles $\left./ \mathrm{mL}\right)$ were suspended in $800 \mu \mathrm{l}$ of $37^{\circ} \mathrm{C}$ PBS in a disposable polystyrene cuvette (One-way transmission factor at $1.0 \mathrm{MHz}=93 \pm 2 \%(\mathrm{n}=10)$; thus, attenuation $\cong 7 \%$ ) (FisherBrand, Thermo Fisher Scientific, Waltham, MA, USA). The unfocused 1.0 MHz transducer was shallowly submerged in the PCCA suspension and set to transmit the same ultrasound pulses that were used for transepithelial delivery (Table 1) with the only difference being that the pulse repetition frequency was significantly alters the interpretation of our results, as discussed in subsequent sections. 
A separate, single element, spherically focused transducer (7.5 MHz nominal frequency, measured center frequency $=8.740 \mathrm{MHz}$, diameter $=19 \mathrm{~mm}$, focal distance $=50 \mathrm{~mm},-6 \mathrm{~dB}$ bandwidth $=1.270$ MHz) (IL0706HP, Valpey Fisher Corp., Hopkinton, MA, USA) was arranged perpendicular to the transmit transducer with its focus positioned within the PCCA solution. Acoustic signals received by this transducer were passed through a receive amplifier (BR-640A, RITEC, Inc., Warwick, RI, USA) with 27 $\mathrm{dB}$ gain and a $500 \mathrm{kHz}$ high pass filter. Signals were subsequently digitized using a 12-bit analog-todigital conversion card with a sampling rate of $200 \mathrm{MHz}$ (GaGe model \#CSE1222, DynamicSignals LLC, Lockport, IL, USA) installed in a computer (Dell, Round Rock, TX, USA) running a custom acquisition program (LabVIEW, National Instruments Corp., Austin, TX, USA).

We saved signals over a total of 30 seconds to monitor the dynamics of cavitation energy generated from the acoustically-stimulated PCCAs. Ultrasound was transmitted continuously throughout the 30second period; however, signals were saved intermittently to reduce data to a manageable size. Each second, 20 individual signals (i.e., $10 \mathrm{~ms}$ of data) were saved followed by a $990 \mathrm{~ms}$ saving delay. This was repeated every second for a total of 30 seconds.

All saved data was post-processed using an adaptation of our previously described post-processing analysis script in MATLAB (MathWorks Inc., Natick, MA, USA) to quantify stable and inertial cavitation doses (Fix, et al. 2017). Briefly, for each individual radiofrequency line, the PCCA signal was selected by applying a $30 \mu \mathrm{s}$ window from the point corresponding to the beginning of the acoustic pulse. Fast Fourier transform was used to convert individual time-domain signals to the frequency domain in order to estimate the level of stable and inertial cavitation. For stable cavitation, the area under curve (AUC) of the second harmonic component was calculated considering a spectral window from 1.9 to $2.1 \mathrm{MHz}$. For inertial cavitation, the broadband emission was quantified by calculating the AUC of frequency content ranging 
cavitation doses were then calculated by normalizing AUC values obtained for PCCAs with those calculated for a PBS-filled cuvette (without PCCAs) exposed to the same acoustic parameters. This was repeated for three independent vials of PCCAs; average cavitation doses (relative AUC) with inter-vial standard deviation are reported. Finally, average of relative AUC for stable and inertial cavitation are plotted over the 30 second period, and total cavitation dose for each was calculated by taking the area under these curves.

\section{Statistical analyses}

All statistical analyses were performed in GraphPad Prism 6 (GraphPad Software, Inc., La Jolla,

CA, USA). P-values of $<0.05$ were considered statistically significant, and data are presented as average \pm standard deviation throughout this work.

To evaluate which ultrasound parameters resulted in statistically significant dextran delivery, we performed a two-way repeated-measures ANOVA with respect to time and acoustic treatment. At each timepoint, the mean of each treatment group was compared to the mean of the sham control at the same time point via Dunnett's multiple comparisons testing. The same statistical procedure was applied to analyze changes in TEER values over time in various ultrasound treatment groups vs. time pointrespective sham controls.

Cumulative dextran delivery achieved at 72 hours was analyzed via an additional two-way ANOVA with respect to acoustic pressure and pulse length. The purpose of this test was to evaluate the extent to which dextran delivery is affected by these factors (i.e. the percent of variability in dextran delivery that can be attributed to pressure vs. number of cycles). Following this two-way ANOVA, two multiple comparisons tests were performed. First, a Sidak multiple comparison test was used to compare mean delivery efficiency between 20 and 40 cycle cases at each pressure. Second, a Tukey multiple 
comparison test was used to compare mean delivery efficiency between all rarefactional pressures separately for the 20 and 40 cycle cases.

The relationship between monolayer disruption and percent dextran delivery was assessed by

314 calculating the correlation between time to monolayer recovery and cumulative percent dextran delivery at the 72-hour time point. Spearman correlation coefficients (r) and p-values are reported. cavitation dose, (2) total inertial cavitation dose, and (3) the sum of total stable and inertial cavitation. This was done separately for 20-cycle and 40-cycle conditions. Again, goodness-of-fit $\left(\mathrm{r}^{2}\right)$ and p-values are reported.

\section{RESULTS AND DISCUSSION}

\section{$322 \quad$ PCCA characterization}

The PCCA formulation was characterized by a polydisperse size distribution with an average concentration of $(1.4 \pm 0.2) \times 10^{12}$ particles $/ \mathrm{mL}$ and mean particle size of $170 \pm 20 \mathrm{~nm}(\mathrm{n}=3$ independent vials) (Figure 2A). Figure 2B shows representative optical microscopy images captured before $(\mathrm{t}=-2 \mathrm{~ms})$ and after $(\mathrm{t}=8 \mathrm{~ms})$ a single 20 -cycle ultrasound pulse at $\mathrm{t}=0 \mathrm{~ms}$. We found that PCCAs consistently vaporized into microbubbles at and above a peak negative pressure of $300 \mathrm{kPa}$, but showed little to no activation at lower pressures (at 1.0 MHz), which is consistent with our previous findings (Fix, et al. 2017). Qualitatively, we observed an increase in the number of bubbles generated with a single ultrasound pulse as pressure was increased from $300 \mathrm{kPa}$ to $600 \mathrm{kPa}$ (Figure 2A). From these experiments, we concluded that the pressure threshold for PCCA vaporization is between $100-300 \mathrm{kPa}$ at $1.0 \mathrm{MHz}$, and therefore pressures at or above $300 \mathrm{kPa}$ were used for all subsequent experiments.

\section{Ultrasound-mediated FITC-dextran delivery through epithelial monolayers}


As expected, $70 \mathrm{kDa}$ dextran was not able to penetrate sham-treated Caco-2 monolayers over the $72 \mathrm{hr}$ incubation period, confirming that effective barrier function had been established in our model (Figure 3A). Similarly, we did not observe appreciable delivery through control monolayers treated with (1) PCCAs only (no ultrasound) or (2) ultrasound only (no PCCAs, ultrasound stimulation at the highest setting of $600 \mathrm{kPa}$ and 40 cycles) (Figure 3A). This indicated that neither our agents nor ultrasound alone altered monolayer integrity with the parameters used in our study. Correspondingly, TEER values for all control groups remained well above the previously chosen threshold for an intact monolayer (560

$\mathrm{Ohm} \cdot \mathrm{cm}^{2}$ ) throughout the 72-hour observation period, further demonstrating that control treatments did not significantly alter membrane integrity (Figure 4A). Neither PCCAs alone nor ultrasound alone resulted in statistically significant changes in TEER values at any time point compared to the time-point respective sham controls (Table 2).

When ultrasound was combined with PCCAs we were able to achieve significant delivery (vs. time-point respective sham controls) of both dextran sizes with select parameter combinations (Figure 3B and 3C). When substantial delivery was achieved, it most often reached statistically significant levels between 8-24 hours post-treatment with cumulative percent delivery increasing slightly thereafter. We note that we do not see appreciable dextran accumulation before the $8 \mathrm{hr}$ time point in any case, and we speculate that the lag time may be due to the diffusional characteristics of the dextran molecules. Future experiments will look into the origin of this lag time with greater detail. In general, the dextran delivery results correspond with TEER values indicating significant monolayer disruption (Figure 4 and Table 2). For all ultrasound-stimulated PCCA treatments, we observed an immediate and statistically significant reduction in TEER, with all groups characterized by average TEER values in the range of 200-280 Ohm $\cdot \mathrm{cm}^{2}$ approximately 5 minutes post-treatment (Figure 4 and Table 2). TEER values tended to recover 
the only conditions that did not show $100 \%$ recovery of all monolayers by the 72 -hour time point. For the $300 \mathrm{kPa}$ treatment groups, we achieved $33 \%$ and 50\% recovery for the 20 and 40 cycle cases, respectively. Treatment groups diverged with respect to monolayer recovery time. To investigate this further, we interpolated "time to monolayer recovery" for each treatment (i.e., time for a monolayer to regain a TEER value $\geq 560 \mathrm{Ohm} \cdot \mathrm{cm}^{2}$ ). This summary value, which captures the monolayer recovery dynamics, correlated well with the cumulative percent dextran delivered $(\mathrm{r}=0.8475, \mathrm{p}<0.0001)$ (Figure 5). Biologically, the main factor dictating dextran delivery efficiency appears to be how long the monolayers remain leaky.

In terms of acoustic parameters, rarefactional pressure has a greater influence on dextran delivery outcome than pulse length. To test this observation statistically, we performed a two-way ANOVA to quantify the relative contributions of acoustic pressure and pulse length to overall variation in cumulative dextran delivery $(72 \mathrm{hr}$ time point $)$. We found that pressure $(\mathrm{p}=0.0002)$ but not pulse length $(\mathrm{p}=0.1476)$ was a significant source of variability in percent dextran delivery, with pressure accounting for $53.5 \%$ of the total variation compared to $3.5 \%$ attributed to pulse length. This can be assessed visually in Figure 6 , where the cumulative percent delivery varies substantially with respect to pressure but remains fairly consistent between pulse lengths. Indeed, multiple comparison testing showed that differences in cumulative dextran delivery between 20 and 40 cycle conditions were statistically insignificant at each rarefactional pressure.

The most interesting and unexpected finding was that the $300 \mathrm{kPa}$ exposure conditions consistently provided significantly greater dextran delivery compared to higher-pressure groups, while the higherpressure conditions $(400,500$, and $600 \mathrm{kPa})$ provided similar results (statistically insignificantly different) (Figure 6). These findings are counter to our initial hypothesis that as acoustic energy was increased, a greater proportion of PCCAs would vaporize into microbubbles and cavitation dose would be more 
pronounced, together leading to more dramatic biological effects and ultimately greater dextran delivery efficiency. We hypothesize that these unexpected findings may be explained by differences in cavitation persistence between ultrasound exposure conditions, as is explored in the subsequent section.

\section{Persistence of cavitation over 30 second exposures}

In our experiments, as would be the case for GI drug delivery applications, the same sample of PCCAs were stimulated for the duration of the ultrasound exposure time (30 seconds in this study). Therefore, at high pressure, PCCAs may be rapidly converted to microbubbles but also subsequently destroyed via inertial cavitation. This would limit the effective cavitation duration to a fraction of the ultrasound on time (30 s). Conversely, if PCCAs were being vaporized more slowly and were able to persist for longer at lower pressures, the effects of microbubble cavitation would have been felt by the cells for a longer period of time, enhancing the biological effect.

To test this hypothesis, we quantified stable and inertial cavitation dose generated from acoustically-stimulated PCCAs in vitro over the course of 30 seconds. All parameters matched those of our dextran delivery experiments except for pulse repetition frequency, which was reduced to $2000 \mathrm{~Hz}$.

For all acoustic exposure conditions (300-600 kPa with 20 and 40 cycle pulse lengths), we observed substantial stable and inertial cavitation immediately after the ultrasound transmission was initiated (Figure 7A-D). For 20 cycle pulse lengths, there is a clear separation in cavitation dynamics with respect to rarefactional pressures, with lower pressures resulting in more sustained stable and inertial cavitation compared to the higher pressures. The lower pressures (especially $300 \mathrm{kPa}$ ) provide sustained stable and inertial cavitation activity over the entire 30 second exposure, while cavitation dose decreases dramatically within the first $15 \mathrm{~s}$ for the higher pressures (especially $600 \mathrm{kPa}$ ) (Figure 7A \& 7C). This trend is also observed for stable cavitation dose with respect to pressure at 40 cycles (Figure 7B). This is less pronounced for inertial cavitation dose at 40 cycles, as cavitation dose decreases more rapidly for all 
pressures (Figure 7D). In general, these data demonstrate that both peak rarefactional pressure and pulse length influence cavitation persistence for PCCAs, as has previously been reported for microbubbles (Pouliopoulos, et al. 2016). The lower pulse repetition frequency used for these experiments compared to the dextran delivery experiments may have biased results slightly. At a higher pulse repetition frequency, cavitation dose would be expected to decrease more rapidly for all conditions, thus this effect is likely more pronounced in our cell experiments at higher pulse repetition frequency.

In general, we hypothesize that two main factors contribute to the inverse trend between total cavitation dose and pressure. First, we qualitatively observed that PCCA vaporization efficiency increases with increasing pressure. This can be observed in the representative images shown in Figure 2, where more bubbles are generated after a single ultrasound pulse at $600 \mathrm{kPa}$ vs. $300 \mathrm{kPa}$. This qualitative finding is supported by previous work showing that relative PCCA vaporization efficiency increases with increasing pressure (Reznik, et al. 2013, Wu, et al. 2018). As a result, it will take longer to vaporize all PCCAs in the sample with lower pressures, contributing to sustained cavitation activity over the 30 -second exposure time. Secondly, as pressure increases, we believe that generated microbubbles will be destroyed more rapidly, ultimately limiting microbubble lifetime and cavitation persistence. A similar inverse trend between generated microbubble stability/survival and pressure has previously been described by Reznik et al. (Reznik, et al. 2013).

To quantitatively analyze the trends in acoustic cavitation with respect to pressure, we first calculated the cumulative stable cavitation dose (SCD) and cumulative inertial cavitation dose (ICD) by calculating the area under each of the cavitation curves over the $30 \mathrm{~s}$ ultrasound exposure. This provided single values summarizing the total SCD and ICD generated by each condition. We also summed these cumulative SCD and ICD values to provide an estimate of the overall cavitation dose generated per condition (SCD+ICD). Linear regressions were performed between each of these summary values and 
acoustic pressure (Figure 7E-F). For the 20 cycle cases, we find statistically significant inverse trends between pressure and total SCD+ICD and total SCD $(p=0.0007-0.0100)$. For the 40 cycle cases, all three summary cavitation metrics were found to significantly and inversely trend with pressure $(\mathrm{p}=0.0049-0.0100)$. For both pulse lengths, $300 \mathrm{kPa}$ qualitatively provided the greatest cumulative SCD, ICD and SCD+ICD compared to all higher-pressure groups, which corresponds with our finding that $300 \mathrm{kPa}$ results in the greatest dextran delivery efficiency.

\section{$432 \quad$ Limitations}

Total cavitation dose alone cannot predict dextran delivery efficiency as evidenced by the discrepancy we find between 20 and 40 cycle conditions: dextran delivery outcomes are comparable across pulse lengths, while total cavitation doses were in general lower for 40 cycle conditions compared to respective 20 cycle conditions. For the 20 cycle conditions, we find a significant correlation between cumulative dextran delivery and (1) total SCD (Pearson $r=0.9624, p=0.0376$ ) and (2) total SCD+ICD (Pearson $\mathrm{r}=0.9638, \mathrm{p}=0.0362$ ). However, for the 40 cycle cases we do not find significant correlations between dextran delivery and any of the total cavitation metrics. This discrepancy may be due to various confounding variables that were not captured by the total cavitation dose metric. For instance, we hypothesize that peak cavitation dose and the balance between stable and inertial cavitation may be important variables contributing to biological outcome.

Furthermore, acoustic pressure and pulse length are known to influence the stability and size distribution of microbubbles generated by PCCA vaporization (Reznik, et al. 2013, Sheeran, et al. 2013), and differences in microbubble populations may have influenced dextran delivery efficiency. The proportion of small microbubbles generated by PCCA vaporization has previously been show to increase with increasing acoustic pressure (Fix, et al. 2017, Sheeran, et al. 2013), which is attributed to the inverse relationship between vaporization pressure threshold and PCCA size (Sheeran and Dayton 2012, Sheeran, 
et al. 2013). Additionally, the likelihood of microbubble fusion (i.e., generation of larger microbubbles) increases with increasing pulse length (Sheeran, et al. 2013). Furthermore, differences in bubble aggregation and coalescence due to secondary Bjerknes forces may alter the number and size of bubbles between treatment groups. These expected differences in microbubble size distribution as a function of acoustic excitation parameters may influence monolayer disruption efficiency.

After ultrasound-mediated monolayer disruption, TEER value analysis indicated that it typically took 24-48 hours for the epithelial cells to regain their integrity. While recovery mechanism was not explicitly studied in this work, this rather long recovery time may indicate that recovery is due to cell repopulation and growth rather than resealing of transiently opened tight junctions between cells. A limitation of this study is that we did not quantify cell death after treatment. Furthermore, not all monolayers treated with the $300 \mathrm{kPa}$ conditions recovered within the 72-hour observation window. We do not claim that we have found optimal acoustic parameters for transepithelial delivery in vivo. Rather, this study provides an encouraging proof of principle demonstration that transepithelial delivery is feasible with ultrasound stimulated PCCAs and supports further optimization of acoustic parameters to maximize transient disruption while minimizing recovery time and cell death.

It is important to note that cell-based assays of intestinal permeabilization are known to overestimate damage compared to the what would be observed in viable intestinal tissue (in the context of chemically induced permeability enhancement) (McCartney, et al. 2016, Petersen, et al. 2013). This is attributed to the lack of complete intestinal repair mechanisms and protective mucus in simple cell culture assays. As a result, any future optimization of acoustic parameters should be carefully validated (e.g., through the in situ intestinal perfusion (Escribano, et al. 2012)) to ensure that the chosen parameters are effective and safe when working with viable intestinal tissue. One of the greatest safety concern for intestinal barrier disruption in vivo is the risk of facilitating absorption of harmful bystanders such as 
bacteria, viruses, and toxins (McCartney, et al. 2016). This risk should be carefully evaluated and minimized upon in vivo translation.

\section{Future directions - potential for ultrasound-mediated GI drug delivery with PCCAs}

475 The data presented herein represent a first step towards the ultimate goal of ultrasound-mediated GI drug delivery with PCCAs, and the finding that lower pressure results in greater delivery efficiency is important for successful in vivo translation. In the in vivo setting, we envision co-administering a medicated enema with a solution of PCCAs. Subsequently, an endoscopic ultrasound probe would be inserted and used to stimulate PCCAs for GI permeabilization. Without natural replenishment of the PCCAs (e.g., through blood flow), achieving persistent cavitation by limiting the destruction of generated microbubbles will be of utmost importance. enhance drug delivery through vascular barriers. For vascular disruption, microbubbles are administered intravenously, and blood flow provides continuous replenishment of intact microbubbles. Indeed, delivery efficiency has been shown to increase with increasing pressure for this application (Chen and Konofagou 2014, Liu, et al. 2012, Wang, et al. 2015), and microbubble reperfusion into the focal zone can be achieved by alternating sonication with rest time (Mullick Chowdhury, et al. 2016, Wang, et al. 2015) or theoretically by using short pulses and long pulse repetition periods (Shamout, et al. 2015). However, using high pressures for vascular permeabilization is associated with an increased risk of undesirable bioeffects (Baseri, et al. 2010), pointing to another motivation for using reduced pressures for any drug delivery application. To this end, Pouliopoulos and colleagues have demonstrated the value of redefining 492 the acoustic pulses typically used for vascular permeabilization to provide sustained, controllable, and 493 safe cavitation activity with low pressures and short pulse lengths (Pouliopoulos, et al. 2014, Pouliopoulos, 494 et al. 2016). 
The likelihood that ultrasound will induce cavitation is related to the mechanical index $(\mathrm{MI}=$ peak rarefactional pressure divided by center frequency) of the transmitted pulse. The United States Food and Drug Administration stipulates that MI cannot exceed 1.9 for clinical diagnostic ultrasound, which is intended to prevent cavitation-mediated biological effects in the absence of exogenous contrast agents. Therefore, when ultrasound-mediated GI permeabilization is performed in the absence of contrast agents, MIs above this threshold (MI 2.2 - 3.3) are often employed to generate the cavitation activity necessary for permeabilization (Schoellhammer, et al. 2017, Schoellhammer, et al. 2017, Schoellhammer, et al. 2015). (Note: these MIs were estimated based on the reported acoustic intensities for ex vivo experiments, assuming plane wave transmission, an acoustic impedance of water of $1.48 \times 10^{6} \mathrm{~kg} \cdot \mathrm{m}^{-2} \cdot \mathrm{s}^{-1}$, negligible tissue attenuation, and that the reported intensity represented instantaneous acoustic intensity.) While these protocols have generally been described as safe in preclinical studies (Schoellhammer, et al. 2017, Schoellhammer, et al. 2015), cavitation-mediated biological effects could be induced in tissues beyond the GI epithelium. Conversely, we were able to achieve efficient drug delivery at a mechanical index of $0.3(300 \mathrm{kPa}$ at $1.0 \mathrm{MHz})$, which is well below the FDA limit of $\mathrm{MI}=1.9$, indicating that biological effects will likely only be induced in areas with direct contact to the contrast agents. This claim is supported by the absence of monolayer disruption (as evidenced by stably high TEER values and impermeability to dextran) when cells were stimulated with ultrasound alone in our experiments. This offers an opportunity

512 for improved treatment localization and the potential for enhanced safety compared to ultrasoundmediated GI drug delivery in the absence of contrast agents.

In the GI space, we believe low boiling point PCCAs will provide greater success than standard microbubbles. Unlike microbubble contrast agents, the small size of PCCAs may allow permeation through the GI mucus mesh and permeabilization of the underlying GI epithelium. In future studies, we 
will test the penetration of PCCAs through mucus and optimize acoustic parameters for GI drug delivery in a model that more accurately captures the in vivo setting (e.g., in situ intestinal perfusion).

The use of ultrasound for drug delivery offers an opportunity for simultaneous image guidance. This will be particularly important for GI drug delivery applications where site-specific (e.g., immunotherapy delivery to a colon tumor) rather than global GI drug delivery is desired. Ultrasound image guidance could be achieved in one of two ways. First, it may be possible to achieve ultrasound imaging and subsequent drug delivery with clinically available endoscopic ultrasound probes (e.g. with $5 \mathrm{MHz}$ center frequency). A similar approach has been published for externally applied ultrasound for drug delivery to pancreatic tumors with a clinically available machine (Dimcevski, et al. 2016, Kotopoulis, et al. 2013). Perhaps more exciting is the prospect of developing dual frequency endoscopic ultrasound probes that incorporate aligned low frequency elements for optimal drug delivery and high frequency elements for high-resolution ultrasound imaging. The prospect of high-frequency and therefore high-resolution image guidance may be particularly advantageous for small animal studies where anatomical features are difficult to resolve with relatively low-frequency imaging. Dual frequency transducers are currently under development for contrast-enhanced intravascular and intracavity imaging (Kim, et al. 2015, Lindsey, et al. 2017, Wang, et al. 2017), which could be modified for image-guided therapy purposes. A dual frequency approach can also be employed to initiate PCCA phase change at high frequency and detect the unique acoustic signature of PCCA vaporization at low frequency (Arena, et al. 2015). This would offer a method to image the efficiency and duration of PCCA vaporization events during the treatment.

\section{CONCLUSIONS}

We have demonstrated efficient delivery of a macromolecular drug mimic (70 kDa FITC-dextran) 
540 found that the lowest pressure conditions $(300 \mathrm{kPa})$ consistently provided the greatest dextran delivery 541 efficiency (vs. 400-600 kPa), which is explained in part by the observation that cavitation is more 542 persistent during ultrasound exposure at lower pressures. While we believe that persistent cavitation 543 activity is important for achieving efficient epithelial disruption, we have not fully explored other acoustic 544 metrics that may also play a role, such as acoustic radiation force, peak cavitation dose, and variable size 545 distributions of acoustically generated microbubbles. Further experimentation should be conducted to 546 evaluate the relative importance of these variables and their contribution to dextran delivery outcome. 547 Insight gleaned from these experiments will allow for rational and thorough optimization of acoustic 548 parameters for in vivo drug delivery through the GI epithelial barrier.

\section{ACKNOWLEDGEMENTS}

The authors would like to thank Eric Markley for assistance adapting the LabVIEW code used in this study, as well as Catherine Feng and Dr. Nicholas Shaheen for useful discussions on the gastrointestinal drug delivery background relating to this work. Financial support was provided by an NRSA Individual Predoctoral Fellowship (F31CA220970, S.M.F) and the University of North Carolina General Assembly Research Opportunities Initiative: Pharmacoengineering (V.P \& B.P.K). perfluorocarbon agents and a co-founder of Triangle Biotechnology, a company that has licensed this patent. All the other authors declare that they have no competing interests. 


\section{REFERENCES}

561 Apfel RE, Holland CK. Gauging the likelihood of cavitation from short-pulse, low-duty cycle diagnostic ultrasound. Ultrasound Med Biol 1991; 17:179-85.

Arena CB, Novell A, Sheeran PS, Puett C, Moyer LC, Dayton PA. Dual-frequency acoustic droplet vaporization detection for medical imaging. IEEE Trans Ultrason Ferroelectr Freq Control 2015; 62:1623-33.

Barrett KE. Epithelial biology in the gastrointestinal system: insights into normal physiology and disease pathogenesis. The Journal of Physiology 2012; 590:419-20.

Baseri B, Choi JJ, Tung YS, Konofagou EE. Multi-modality safety assessment of blood-brain barrier opening using focused ultrasound and definity microbubbles: a short-term study. Ultrasound in medicine \& biology 2010; 36:1445-59.

Bhatnagar S, Schiffter H, Coussios CC. Exploitation of acoustic cavitation-induced microstreaming to enhance molecular transport. Journal of pharmaceutical sciences 2014; 103:1903-12.

Burgess A, Hynynen K. Drug delivery across the blood-brain barrier using focused ultrasound. Expert Opin Drug Deliv 2014; 11:711-21.

Burgess MT, Porter TM. Acoustic Cavitation-Mediated Delivery of Small Interfering Ribonucleic Acids with Phase-Shift Nano-Emulsions. Ultrasound in medicine \& biology 2015; 41:2191-201.

Chen H, Konofagou EE. The size of blood-brain barrier opening induced by focused ultrasound is dictated by the acoustic pressure. Journal of cerebral blood flow and metabolism : official journal of the International Society of Cerebral Blood Flow and Metabolism 2014; 34:1197-204.

Cone RA. Barrier properties of mucus. Advanced drug delivery reviews 2009; 61:75-85.

Coussios C, Myers R, Kwan J, Lea-Banks H, Mo S, Grundy M, Mannaris C, Duffy M, Paverd C, Carugo D, Coviello C, Stride E, Carlisle R. Cavitation-mediated extravasation and transtumoral drug 
delivery by microstreaming: What role do the gas nuclei and the physical properties of the therapeutic play? The Journal of the Acoustical Society of America 2016; 140:2985-85.

585 Delalande A, Kotopoulis S, Rovers T, Pichon C, Postema M. Sonoporation at a low mechanical index. 586 Bubble Science, Engineering \& Technology 2011; 3:3-12.

Dimcevski G, Kotopoulis S, Bjanes T, Hoem D, Schjott J, Gjertsen BT, Biermann M, Molven A, Sorbye 588 to enhance gemcitabine treatment of inoperable pancreatic cancer. Journal of controlled release : official journal of the Controlled Release Society 2016; 243:172-81.

Escoffre JM, Bouakaz A. Mini-review - Biophysical mechanisms of cell membrane sonopermeabilization: Knowns and unknowns. Langmuir 2018.

Escribano E, Sala XG, Salamanca J, Navarro CR, Regué JQ. Single-pass intestinal perfusion to establish the intestinal permeability of model drugs in mouse. Int J Pharm 2012; 436:472-7.

Fabiilli ML, Haworth KJ, Fakhri NH, Kripfgans OD, Carson PL, Fowlkes JB. The role of inertial cavitation in acoustic droplet vaporization. IEEE transactions on ultrasonics, ferroelectrics, and frequency control 2009; 56:1006-17.

Fix SM, Novell A, Yun Y, Dayton PA, Arena CB. An evaluation of the sonoporation potential of lowboiling point phase-change ultrasound contrast agents in vitro. Journal of therapeutic ultrasound 2017; 5:7.

Goldberg M, Gomez-Orellana I. Challenges for the oral delivery of macromolecules. Nature Reviews Drug Discovery 2003; 2:289.

Kim J, Li S, Kasoji S, Dayton PA, Jiang X. Phantom evaluation of stacked-type dual-frequency 1-3 composite transducers: A feasibility study on intracavitary acoustic angiography. Ultrasonics $2015 ; 63: 7-15$. 
606 Kooiman K, Vos HJ, Versluis M, de Jong N. Acoustic behavior of microbubbles and implications for drug 607 delivery. Advanced Drug Delivery Reviews 2014; 72:28-48.

608 Kooiman K, Vos HJ, Versluis M, de Jong N. Acoustic behavior of microbubbles and implications for drug 609 delivery. Adv Drug Deliv Rev 2014; 72:28-48.

610 Kotopoulis S, Dimcevski G, Gilja OH, Hoem D, Postema M. Treatment of human pancreatic cancer using 611 combined ultrasound, microbubbles, and gemcitabine: a clinical case study. Med Phys 2013; 40:072902.

613 Kudsiova L, Lawrence MJ. A comparison of the effect of chitosan and chitosan-coated vesicles on monolayer integrity and permeability across Caco-2 and 16HBE14o-cells. J Pharm Sci 2008; 97:3998-4010.

Lentacker I, De Cock I, Deckers R, De Smedt SC, Moonen CT. Understanding ultrasound induced sonoporation: definitions and underlying mechanisms. Advanced drug delivery reviews 2014; 72:49-64.

Lindsey BD, Kim J, Dayton PA, Jiang X. Dual-Frequency Piezoelectric Endoscopic Transducer for Imaging Vascular Invasion in Pancreatic Cancer. IEEE transactions on ultrasonics, ferroelectrics, and frequency control 2017; 64:1078-86.

Liu HL, Hsieh HY, Lu LA, Kang CW, Wu MF, Lin CY. Low-pressure pulsed focused ultrasound with microbubbles promotes an anticancer immunological response. Journal of translational medicine $2012 ; 10: 221$

Lo AH, Kripfgans OD, Carson PL, Rothman ED, Fowlkes JB. Acoustic droplet vaporization threshold: effects of pulse duration and contrast agent. IEEE transactions on ultrasonics, ferroelectrics, and frequency control 2007; 54:933-46. 
Martin KH, Dayton PA. Current Status and Prospects for Microbubbles in Ultrasound Theranostics. Wiley interdisciplinary reviews. Nanomedicine and nanobiotechnology 2013; 5.

McCartney F, Gleeson JP, Brayden DJ. Safety concerns over the use of intestinal permeation enhancers: A mini-review. Tissue Barriers 2016; 4:e1176822.

Miller DL, Pislaru SV, Greenleaf JE. Sonoporation: mechanical DNA delivery by ultrasonic cavitation. Somat Cell Mol Genet 2002; 27:115-34.

Moss DM, Curley P, Kinvig H, Hoskins C, Owen A. The biological challenges and pharmacological opportunities of orally administered nanomedicine delivery. Expert Rev Gastroenterol Hepatol 2017:1-14.

Mullick Chowdhury S, Lee T, Willmann JK. Ultrasound-guided drug delivery in cancer. Ultrasonography 2017; 36:171-84.

Mullick Chowdhury S, Wang TY, Bachawal S, Devulapally R, Choe JW, Abou Elkacem L, Yakub BK, Wang DS, Tian L, Paulmurugan R, Willmann JK. Ultrasound-guided therapeutic modulation of hepatocellular carcinoma using complementary microRNAs. Journal of controlled release : official journal of the Controlled Release Society 2016; 238:272-80.

Pereno V, Aron M, Vince O, Mannaris C, Seth A, de Saint Victor M, Lajoinie G, Versluis M, Coussios C, Carugo D, Stride E. Layered acoustofluidic resonators for the simultaneous optical and acoustic characterisation of cavitation dynamics, microstreaming, and biological effects. Biomicrofluidics 2018; 12:034109.

Petersen SB, Nielsen LG, Rahbek UL, Guldbrandt M, Brayden DJ. Colonic absorption of salmon calcitonin using tetradecyl maltoside (TDM) as a permeation enhancer. Eur J Pharm Sci 2013; 48:726-34. 
650 Postema M, van Wamel A, ten Cate FJ, de Jong N. High-speed photography during ultrasound illustrates potential therapeutic applications of microbubbles. Med Phys 2005; 32:3707-11.

652

654

655

656

Pouliopoulos AN, Bonaccorsi S, Choi JJ. Exploiting flow to control the in vitro spatiotemporal distribution of microbubble-seeded acoustic cavitation activity in ultrasound therapy. Physics in medicine and biology 2014; 59:6941-57.

Pouliopoulos AN, Li C, Tinguely M, Garbin V, Tang MX, Choi JJ. Rapid short-pulse sequences enhance the spatiotemporal uniformity of acoustically driven microbubble activity during flow conditions. J Acoust Soc Am 2016; 140:2469.

Qin J, Wang TY, Willmann JK. Sonoporation: Applications for Cancer Therapy. Adv Exp Med Biol 2016; 880:263-91.

Reznik N, Shpak O, Gelderblom EC, Williams R, de Jong N, Versluis M, Burns PN. The efficiency and stability of bubble formation by acoustic vaporization of submicron perfluorocarbon droplets. Ultrasonics 2013; 53:1368-76.

Schoellhammer CM, Chen Y, Cleveland C, Minahan D, Bensel T, Park JY, Saxton S, Lee YL, Booth L, Langer R, Traverso G. Defining optimal permeant characteristics for ultrasound-mediated gastrointestinal delivery. Journal of controlled release : official journal of the Controlled Release Society $2017 ; 268: 113-19$.

Schoellhammer CM, Langer R, Traverso G. Of microneedles and ultrasound: Physical modes of gastrointestinal macromolecule delivery. Tissue Barriers 2016; 4.

Schoellhammer CM, Lauwers GY, Goettel JA, Oberli MA, Cleveland C, Park JY, Minahan D, Chen Y, Anderson DG, Jaklenec A, Snapper SB, Langer R, Traverso G. Ultrasound-Mediated Delivery of RNA to Colonic Mucosa of Live Mice. Gastroenterology 2017; 152:1151-60. 
672 Schoellhammer CM, Schroeder A, Maa R, Lauwers GY, Swiston A, Zervas M, Barman R, DiCiccio AM, Brugge WR, Anderson DG, Blankschtein D,

D, Langer R, Traverso G. Ultrasound-mediated gastrointestinal drug delivery. Science translational medicine 2015; 7:310ra168.

675

Schoellhammer CM, Traverso G. Low-frequency ultrasound for drug delivery in the gastrointestinal tract. Expert Opin Drug Deliv 2016; 13:1045-8.

Sennoga CA, Kanbar E, Auboire L, Dujardin PA, Fouan D, Escoffre JM, Bouakaz A. Microbubblemediated ultrasound drug-delivery and therapeutic monitoring. Expert Opin Drug Deliv 2017; 14:1031-43.

Shamout FE, Pouliopoulos AN, Lee P, Bonaccorsi S, Towhidi L, Krams R, Choi JJ. Enhancement of noninvasive trans-membrane drug delivery using ultrasound and microbubbles during physiologically relevant flow. Ultrasound in medicine \& biology 2015; 41:2435-48.

Sheeran PS, Dayton PA. Phase-Change Contrast Agents for Imaging and Therapy. Current pharmaceutical design 2012; 18:2152-65.

Sheeran PS, Luois S, Dayton PA, Matsunaga TO. Formulation and acoustic studies of a new phase-shift agent for diagnostic and therapeutic ultrasound. Langmuir : the ACS journal of surfaces and colloids 2011; 27:10412-20.

Sheeran PS, Luois SH, Mullin LB, Matsunaga TO, Dayton PA. Design of ultrasonically-activatable nanoparticles using low boiling point perfluorocarbons. Biomaterials 2012; 33:3262-9.

Sheeran PS, Matsunaga TO, Dayton PA. Phase-transition thresholds and vaporization phenomena for ultrasound phase-change nanoemulsions assessed via high-speed optical microscopy. Physics in medicine and biology 2013; 58:4513-34. 
Sheeran PS, Rojas JD, Puett C, Hjelmquist J, Arena CB, Dayton PA. Contrast-enhanced ultrasound imaging and in vivo circulatory kinetics with low-boiling-point nanoscale phase-change perfluorocarbon agents. Ultrasound Med Biol 2015; 41:814-31.

Sirsi S, Borden M. Microbubble Compositions, Properties and Biomedical Applications. Bubble science engineering and technology 2009; 1:3-17.

Srinivasan B, Kolli AR, Esch MB, Abaci HE, Shuler ML, Hickman JJ. TEER measurement techniques for in vitro barrier model systems. J Lab Autom 2015; 20:107-26.

van Breemen RB, Li Y. Caco-2 cell permeability assays to measure drug absorption. Expert opinion on drug metabolism \& toxicology $2005 ; 1: 175-85$.

Wang TY, Choe JW, Pu K, Devulapally R, Bachawal S, Machtaler S, Chowdhury SM, Luong R, Tian L, Khuri-Yakub B, Rao J, Paulmurugan R, Willmann JK. Ultrasound-guided delivery of microRNA loaded nanoparticles into cancer. Journal of controlled release : official journal of the Controlled Release Society 2015; 203:99-108.

Wang Z, Martin KH, Huang W, Dayton PA, Jiang X. Contrast Enhanced Superharmonic Imaging for Acoustic Angiography Using Reduced Form-factor Lateral Mode Transmitters for Intravascular and Intracavity Applications. IEEE transactions on ultrasonics, ferroelectrics, and frequency control 2017; 64:311-9.

Williams R, Wright C, Cherin E, Reznik N, Lee M, Gorelikov I, Foster FS, Matsuura N, Burns PN. Characterization of submicron phase-change perfluorocarbon droplets for extravascular ultrasound imaging of cancer. Ultrasound in medicine \& biology 2013; 39:475-89.

Wu SK, Chu PC, Chai WY, Kang ST, Tsai CH, Fan CH, Yeh CK, Liu HL. Characterization of Different Microbubbles in Assisting Focused Ultrasound-Induced Blood-Brain Barrier Opening. Scientific reports $2017 ; 7: 46689$. 
716 Wu SY, Fix SM, Arena CB, Chen CC, Zheng W, Olumolade OO, Papadopoulou V, Novell A, Dayton PA, Konofagou EE. Focused ultrasound-facilitated brain drug delivery using optimized nanodroplets: vaporization efficiency dictates large molecular delivery. Physics in medicine and biology 2018; 63:035002.

720 Zhao YZ, Lu CT, Li XK, Cai J. Ultrasound-mediated strategies in opening brain barriers for drug brain delivery. Expert Opin Drug Deliv 2013; 10:987-1001. 
724 Figure 1: (Color online) Sonication strategy. Colorectal adenocarcinoma (Caco-2) cells were cultured on permeable Transwell supports. Phase-change contrast agents (PCCAs) and dextran were added to the donor chamber before sonication and samples were collected from the receiving compartment over the following 72 hours post-ultrasound treatment. The twelve-well culture plates were positioned in a custom water bath and coupled to the $37^{\circ} \mathrm{C}$ water below before treatment. A lid with circular holes was used to align the ultrasound transducer in the center of each well at a consistent height above the cells.

Figure 2: (A) PCCA characterization. The size distribution and concentration of PCCAs were characterized using a Nanosight. The PCCA formulation was characterized by a polydisperse size distribution with an average concentration of $(1.4 \pm 0.2) \times 10^{12}$ particles $/ \mathrm{mL}$ and mean particle size of $170 \pm 20 \mathrm{~nm}$ ( $\mathrm{n}=3$ independent vials). (B) Acoustic PCCA vaporization. Representative high speed optical microscopy images showing PCCA vaporization as a function of rarefactional pressure. Considerable PCCA vaporization is observed at and above $300 \mathrm{kPa}$, with the number of generated microbubbles increasing with increasing pressure. Scale bar $=10 \mu \mathrm{m}$

Abbreviations: PCCA - phase change contrast agent; kPa - kilopascal

Figure 3: Dextran delivery through Caco-2 monolayers. Dextran delivery is presented as a percentage of the maximum dextran mass that would have been found in the receiving compartment if equilibrium had been achieved between the donor and receiver Transwell compartments (i.e. if there was no barrier between chambers). (A) Negligible amounts of the $70 \mathrm{kDa}$ dextran permeated through control treated monolayers over the 72-hour incubation period. When monolayers were sonicated in the presence of PCCAs, significant delivery was achieved with select acoustic parameters (B and C). Stars indicate the results of Dunnett's multiple comparison testing at each timepoint comparing mean percent dextran 
747 delivery of each group to that of the sham control for that timepoint after two-way ANOVA. Data is 748 presented as mean \pm SD. A total of $n=3$ replicates were performed for all conditions except the $300 \mathrm{kPa}-40$ 749 cycle condition, which was repeated $n=6$ times. The three experimental replicates were performed on 750 independent 12-well Transwell plates.

$751 \quad$ Key: $* \mathrm{p} \leq 0.05 ; * * \mathrm{p} \leq 0.01 ; * * * \mathrm{p} \leq 0.001 ; * * * * \mathrm{p} \leq 0.0001$

Figure 4: (Color online) Transepithelial resistance (TEER) values before and after monolayer sonication. Control treated cells did not show any significant change in TEER values after manipulation, and TEER values remained above $560 \mathrm{Ohm} \cdot \mathrm{cm}^{2}$ (green dashed line) throughout the 72-hour observation period, indicating maintained monolayer integrity (A). For all monolayers treated with ultrasound and PCCAs, a significant decrease in TEER values was recorded immediately ( $\sim 5 \mathrm{~min})$ after treatment (B and C). Most of these monolayers recovered (regained TEER values $\geq 560 \mathrm{Ohm} \cdot \mathrm{cm}^{2}$ within $24-48$ hours, with the exception of some monolayers treated with $300 \mathrm{kPa}$ and either 20 or 40 cycle pulse lengths. Data is presented as mean \pm SD. A total of $n=3$ replicates were performed for all conditions except the $300 \mathrm{kPa}-40$ cycle condition, which was repeated $n=6$ times. The three experimental replicates were performed on independent 12-well Transwell plates.

Figure 5: Correlation between cumulative dextran delivery and time to monolayer recovery. A significant correlation was found between percent dextran delivery at 72 hours and interpolated time to monolayer recovery. 
769 Figure 6: Influence of pulse length and rarefactional pressure on overall dextran delivery outcome.

770 Dextran delivery efficiency was comparable for 20 cycle and 40 cycle groups at each rarefactional 771 pressure (statistically insignificantly different). The $300 \mathrm{kPa}$ conditions consistently provided the greatest 772 dextran delivery outcomes compared to all higher-pressure conditions. Higher pressure conditions (400 $773600 \mathrm{kPa}$ ) provided similar delivery outcomes to each other. Statistical comparisons between pressures are 774 shown with red dashed lines and \# symbols for the 20-cycle case and with blue dotted lines and $*$ symbols 775 for the 40-cycle case.

$776 \quad$ Key: * and $\# \mathrm{p} \leq 0.05 ; * * * \mathrm{p} \leq 0.001$

Figure 7: (Color online) Generation of stable and inertial cavitation and trends with rarefactional pressure. (A-D) Acoustic stimulation of PCCAs resulted in the generation of substantial stable cavitation (SCD) and inertial cavitation (ICD) for all acoustic conditions tested. The persistence of this cavitation over 30 seconds varied between conditions. In general, SCD and ICD was most persistent at the lowpressure conditions (300 or $400 \mathrm{kPa}$ ) with short pulse length (20 cycles). Total SCD and ICD dose was calculated as the area under the cavitation curves over 30 seconds. (E) For the 20 cycle conditions, we found significant inverse trend between rarefactional pressure and total SCD+ICD dose and SCD dose. (F) This trend held true for SCD+ICD, SCD and ICD doses for the 40 cycle conditions. All data presented as mean $\pm \mathrm{SD}$ with $\mathrm{n}=3$ replicates. 
Table 1: Summary of conditions tested. Summary of the acoustic conditions tested for ultrasoundmediated Caco-2 permeabilization with PCCAs, including the number of experimental replicates performed.

Abbreviations: PCCA - phase change contrast agent; kPa - kilopascal; kDa - kilodalton; US ultrasound; $\mathrm{Y}$ - yes; $\mathrm{N}$ - no; NA - not applicable

Table 2: Statistics describing transepithelial resistance (TEER) values before and after monolayer

812 sonication. Stars indicate the results of Dunnett's multiple comparison testing at each timepoint 813 comparing mean TEER value of each group to that of the sham control for that timepoint after two-way

\section{ANOVA.}

$815 \quad$ Key: $* \mathrm{p} \leq 0.05 ; * * \mathrm{p} \leq 0.01 ; * * * \mathrm{p} \leq 0.001 ; * * * * \mathrm{p} \leq 0.0001$

816 Abbreviations: PCCA - phase change contrast agent; kPa - kilopascal; US - ultrasound; cyc - cycles.

\begin{tabular}{|c|c|c|c|c|c|c|c|}
\hline \multirow[b]{3}{*}{ Group } & \multicolumn{7}{|c|}{$\begin{array}{l}\text { Significantly different than respective sham } \\
\text { control? }\end{array}$} \\
\hline & \multicolumn{7}{|c|}{ Time point (hours) } \\
\hline & pre & $\mathbf{0}$ & 3 & 8 & 24 & 48 & 72 \\
\hline US only & ns & ns & ns & ns & ns & ns & ns \\
\hline
\end{tabular}




\begin{tabular}{|l|l|l|l|l|l|l|l|}
\hline $\mathrm{PCCA}$ only & $\mathrm{ns}$ & $\mathrm{ns}$ & $\mathrm{ns}$ & $\mathrm{ns}$ & $\mathrm{ns}$ & $\mathrm{ns}$ & $\mathrm{ns}$ \\
\hline $600 \mathrm{kPa}-20 \mathrm{cyc}$ & $\mathrm{ns}$ & $* * * *$ & $* * * *$ & $* * * *$ & $* * *$ & $\mathrm{~ns}$ & $\mathrm{~ns}$ \\
\hline $500 \mathrm{kPa}-20 \mathrm{cyc}$ & $\mathrm{ns}$ & $* * * *$ & $* * * *$ & $* * * *$ & $* * * *$ & $* *$ & $\mathrm{~ns}$ \\
\hline $400 \mathrm{kPa}-20 \mathrm{cyc}$ & $\mathrm{ns}$ & $* * * *$ & $* * * *$ & $* * * *$ & $* * * *$ & $* * * *$ & $\mathrm{~ns}$ \\
\hline $300 \mathrm{kPa}-20 \mathrm{cyc}$ & $\mathrm{ns}$ & $* * * *$ & $* * * *$ & $* * * *$ & $* * * *$ & $* * * *$ & $* * * *$ \\
\hline $600 \mathrm{kPa}-40 \mathrm{cyc}$ & $\mathrm{ns}$ & $* * * *$ & $* * * *$ & $* * * *$ & $* * * *$ & $*$ & $\mathrm{~ns}$ \\
\hline $500 \mathrm{kPa}-40 \mathrm{cyc}$ & $\mathrm{ns}$ & $* * * *$ & $* * * *$ & $* * * *$ & $* * * *$ & $* * *$ & $\mathrm{~ns}$ \\
\hline $400 \mathrm{kPa}-40 \mathrm{cyc}$ & $\mathrm{ns}$ & $* * * *$ & $* * * *$ & $* * * *$ & $* *$ & $\mathrm{~ns}$ & $\mathrm{~ns}$ \\
\hline $300 \mathrm{kPa}-40 \mathrm{cyc}$ & $\mathrm{ns}$ & $* * * *$ & $* * * *$ & $* * * *$ & $* * * *$ & $* * * *$ & $* * * *$ \\
\hline
\end{tabular}




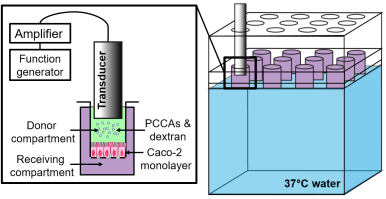


A. Size distribution $\quad$ B. PCCA vaporization

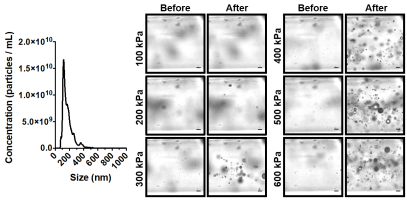




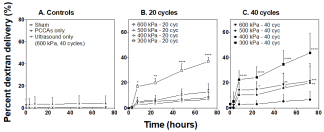


A. Controls

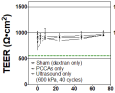

B. 20 oycles

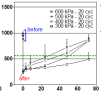

C. 40 grcles

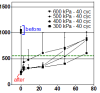

Time (hours) 


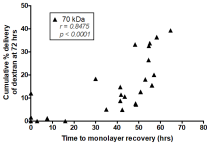




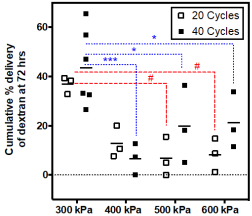

Rarefactional Pressure 
20 Cycles

A

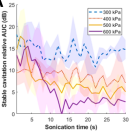

C
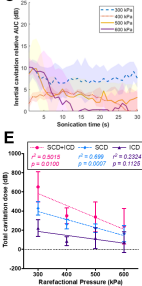

40 Cycles

B

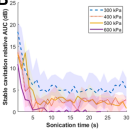

D

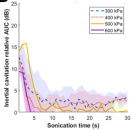

F

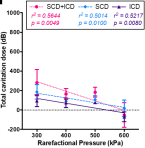

